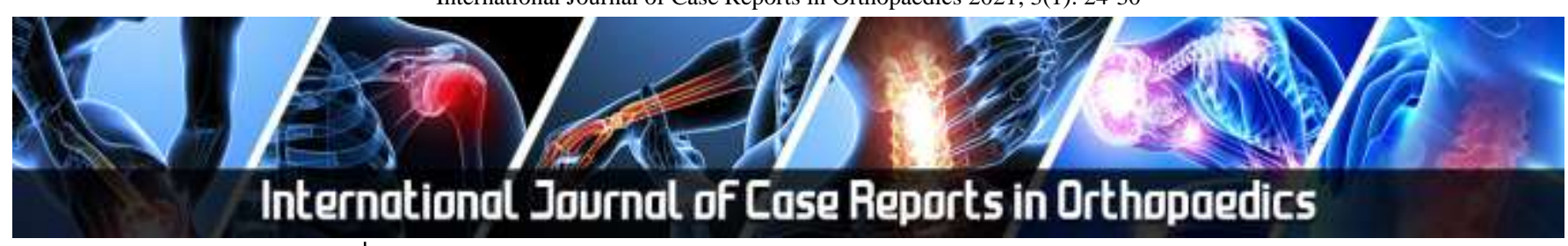

E-ISSN: 2707-8353 P-ISSN: 2707-8345 IJCRO 2021; 3(1): 24-30 Received: 22-11-2020 Accepted: 29-12-2020

Norawee Wainipitapong Department of orthopaedics, Nakhon Pathom hospital, Nakhon Pathom, 73000 , Thailand
Corresponding Author: Norawee Wainipitapong Department of orthopaedics, Nakhon Pathom hospital, Nakhon Pathom, 73000,

Thailand

\section{A computer-assisted navigation technique for complex primary knee arthroplasty with intra-articular bone defect and instability: A case report and literature review}

\section{Norawee Wainipitapong}

DOI: https://doi.org/10.22271/27078345.2021.v3.i1a.45

\begin{abstract}
Correction of a combined severe varus, bone defect, and ligamentous instability in a complex primary knee replacement is a technically demanding procedure. In such instances, constrained or rotatinghinge prosthesis is usually considered. Computer-assisted surgery in extra-articular deformity correction is a widely accepted and advantageous approach, but difficult to apply for intra-articular deformity due to difficulties with defining anatomical references. Our case report describes a patient with severe osteoarthritis, with intra-articular bone defects and ligament instability. We performed a computer-assisted navigation technique by combining gap balancing and measured resection techniques, with a metaphyseal sleeve as a new joint-line reference. We used an unconstrained posterior-stabilized (PS) prosthesis, incorporating Knee Society Scores (KSS): KSS-knee and KSSfunction improved from $0-91$ and 5-85, respectively, at one-month postoperatively. Our technique exhibited good short-term clinical outcomes and may decrease the requirement for constrained or rotating-hinge prostheses in a complex primary case.
\end{abstract}

Keywords: Knee arthroplasty, computer-assisted surgery, navigation, complex primary total knee arthroplasty, intra-articular deformity

\section{Introduction}

Total knee arthroplasty in a complex primary knee is considered a challenging operation; however, multiple techniques have been proposed to achieve good outcomes ${ }^{[1]}$. Patients with severe preoperative deformity, ligamentous instability, and/or marked bone loss occasionally require a varus and valgus, constrained or rotating-hinge design prosthesis, which more than doubles the component revision rate at 10 years, and triples the rate at 20 years, when compared with unconstrained groups ${ }^{[2]}$.

Computer-assisted surgery (CAS) is well established for total knee arthroplasty, and improves the accuracy of both tibial and femoral components, when compared with conventional surgical techniques ${ }^{[3-6]}$. Despite extra-articular deformity, CAS is rarely reported for intra-articular deformity and other complex primary knee cases.

To highlight the CAS benefits in a complex primary knee case with intra-articular deformity, we report the correction of an intra-articular tibial metaphyseal bone defect, severe varus deformity, varus-valgus instability, knee dislocation, and patellofemoral maltracking using computer-assisted navigation. We discuss this complex case here.

\section{Case report}

A 60-year-old female with pain in both knees, deformity, and unable to bear weight over both legs was presented. She had no history of knee injury or infection. After conservative treatment and multiple steroid injections over the previous four years, she displayed progressive deformity and instability in her left knee (Figure 1A). Her range of motion was $10^{\circ}-110^{\circ} ; 40^{\circ}$ varus with $3+$ varus stress test, $1+$ valgus stress test, irreducible anterior knee subluxation, without neurovascular deficit.

Upon radiographic examination, we observed severe tricompartmental osteoarthritis of the knee with anteromedial subluxation, postero-medial tibial bone defect (AORI T2A), and patella subluxation (Figures 1B, 1C). A computed tomography scan revealed similar results with plain radiographs: a $30 \mathrm{~mm}$ tibial bone defect and $6 \mathrm{~mm}$ joint fluid collection. Despite non-visualized ACL and PCL, MCL and LCL were intact using magnetic resonance imaging 
(MRI) (Figures 2A, 2B). Our strategy was to implement CAS incorporating total knee replacement, using either a constrained or hinge knee prosthesis with metaphyseal sleeve, plus tibial stem (P.F.C. ${ }^{\circledR}$ SIGMA® TC3 or SROM ${ }^{\circledR}$ NOILES ${ }^{\mathrm{TM}}$ Rotating Hinge Knee System, DePuy, Inc., Warsaw, IN, USA).

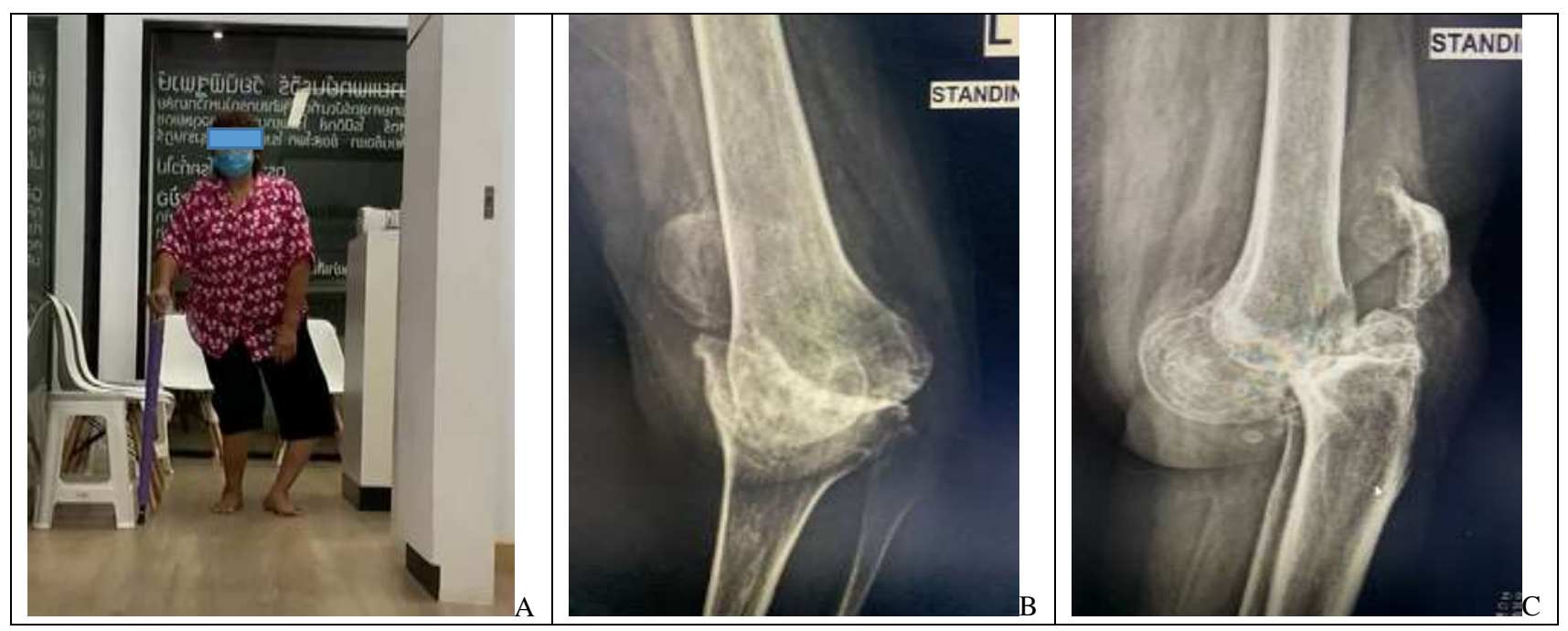

Fig 1: A) Preoperative alignment, B \& C) plain radiographs

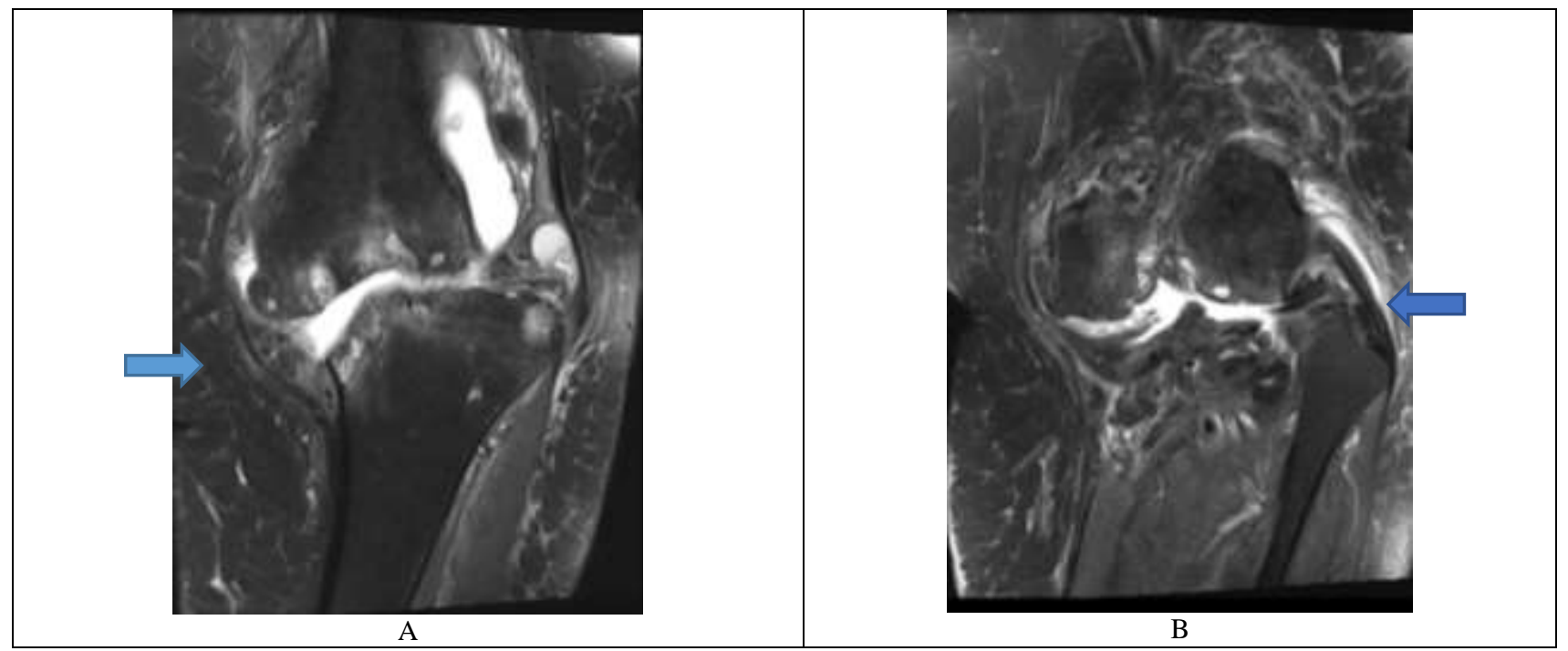

Fig 2: (A \& B) Magnetic resonance imaging (MRI) shows intact MCL and LCL

The knee was exposed via standard medial parapatellar arthrotomy; a prominent osteophyte was removed and patella resurfacing was performed (Figures 3A, 3B). The knee was placed in an appropriate anatomical position before placing the tibial and femur array, and registering local anatomical landmarks (Figure 3C).

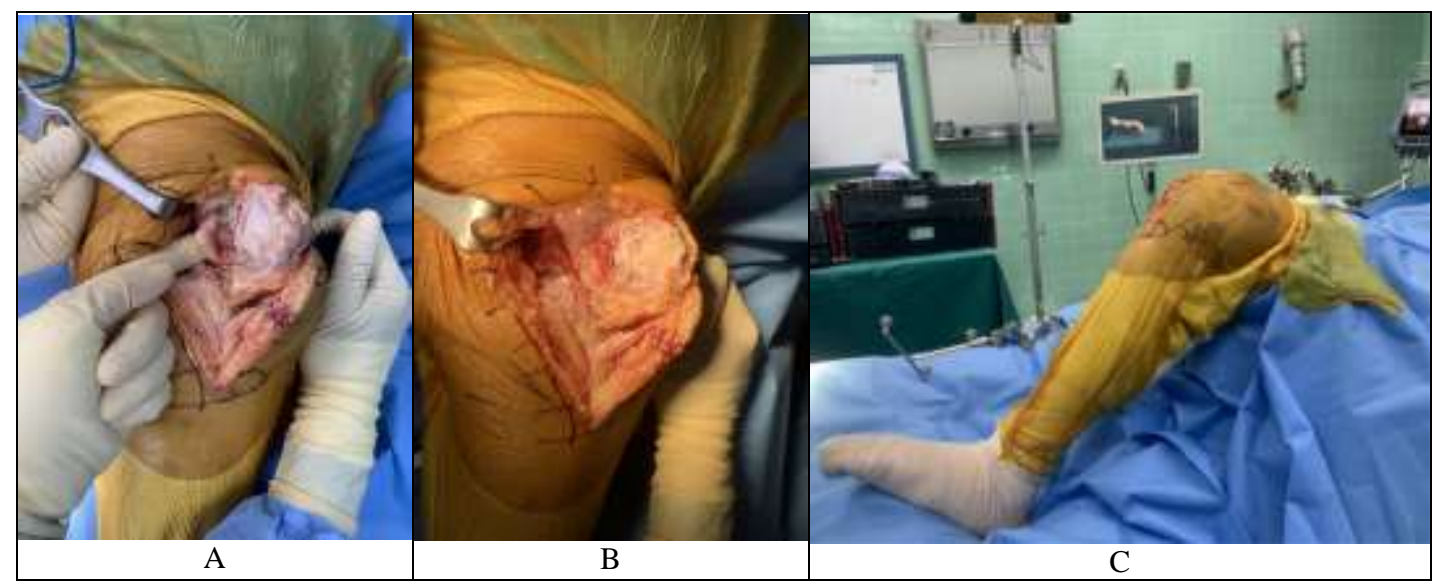

Fig 3: (A \& B) Pre-post patella resurface, C) Knee reduction before CAS registration 
The deepest medial tibial bone defect was measured at 26.5 $\mathrm{mm}$, compared to the lateral side (Figure 4A). We first used the tibial technique; an $8 \mathrm{~mm}$ lateral reference tibial cut, $0^{\circ}$ varus perpendicular to the mechanical axis, and a $2^{\circ}$ posterior slope (Figure 4B). After metaphyseal broaching, a metaphyseal sleeve, stem, and tibial trial were assembled and left in the tibia. A new tibial joint line was then recreated. Navigation was re-registered. The flexion and extension gap were measured by a tensor device, (BalAnswer, DePuy, Inc.) (Figures 4C, 4D).
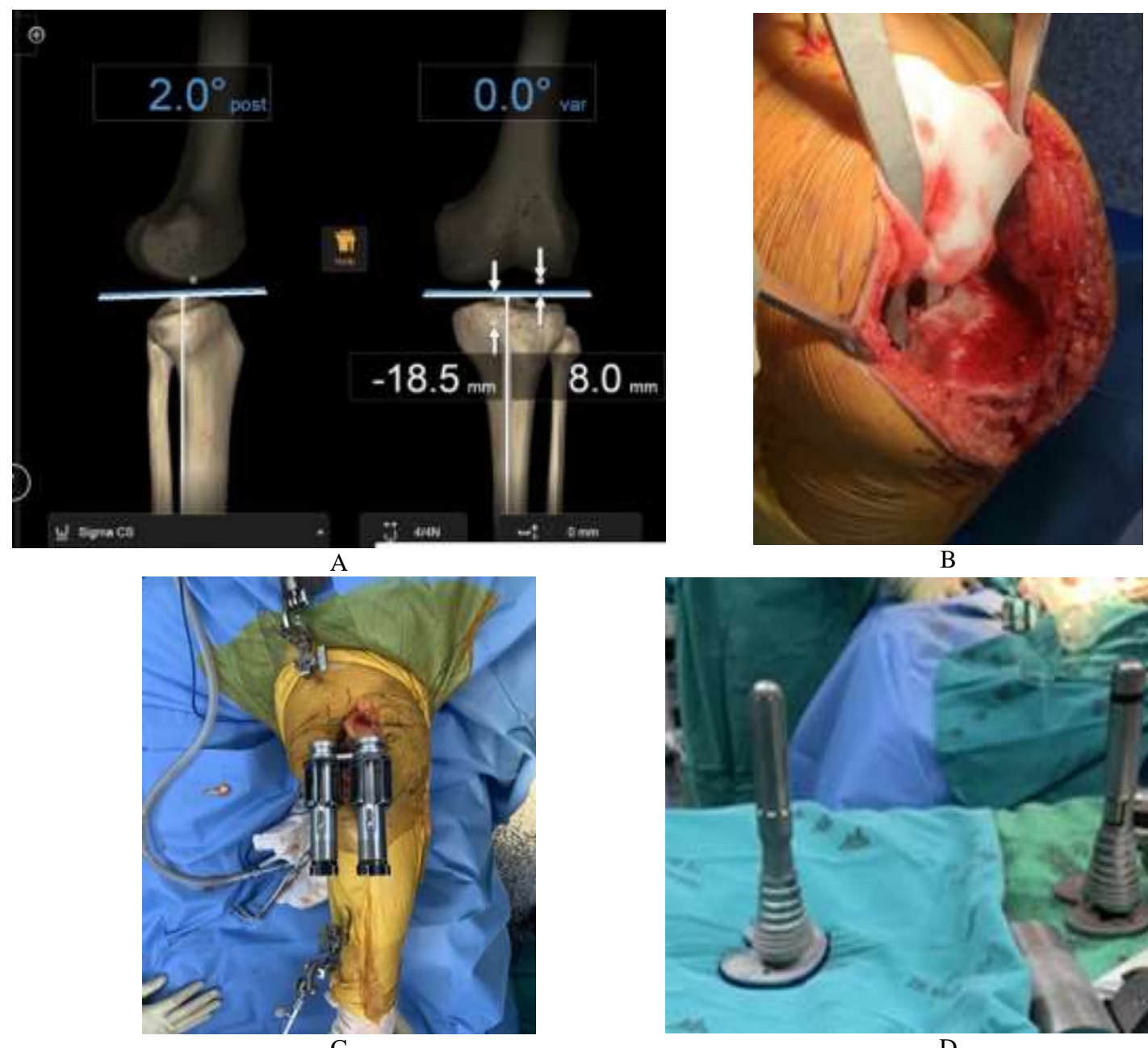

B

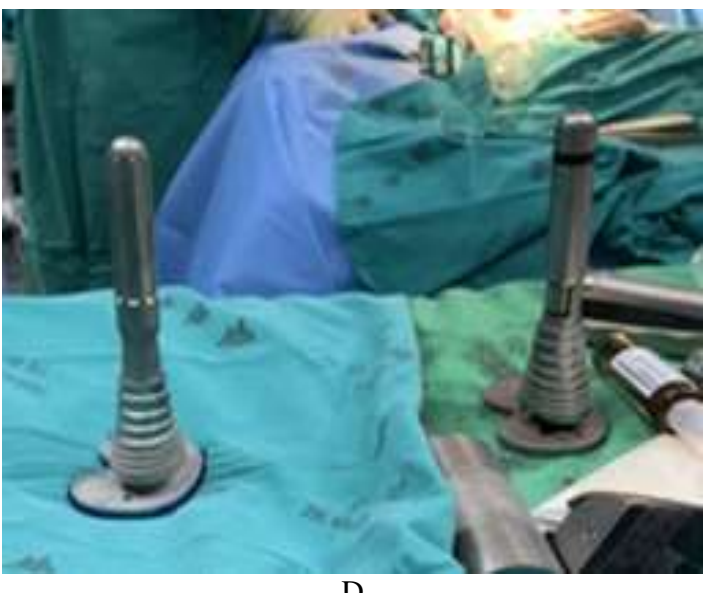

D

Fig 4: A) Cutting reference, B) medial bone defect, C) tensor device, D) trial component.

For the femoral component, rotation and sizing were first determined using computer navigation, and confirmed by trial component, Whiteside's line, and a transepicondylar axis. A size four femoral component was chosen for the procedure. A femoral cutting block was positioned perpendicular to mechanical axis, $9 \mathrm{~mm}$ distal femoral resection. A symmetrical gap was planned by setting a component at $3^{\circ}$ external rotation, $1^{\circ}$ valgus, $2.5^{\circ}$ external rotation and $2.5^{\circ}$ flexion (Figure 5).

After rechecking with CAS, slightly tight extension gap with well-balanced flexion gap was corrected by shift in the distal femoral cutting guide to $10.5 \mathrm{~mm}$ thickness, trial was inserted, pulled-out and lift off test were negative and no residual lateral laxity. 


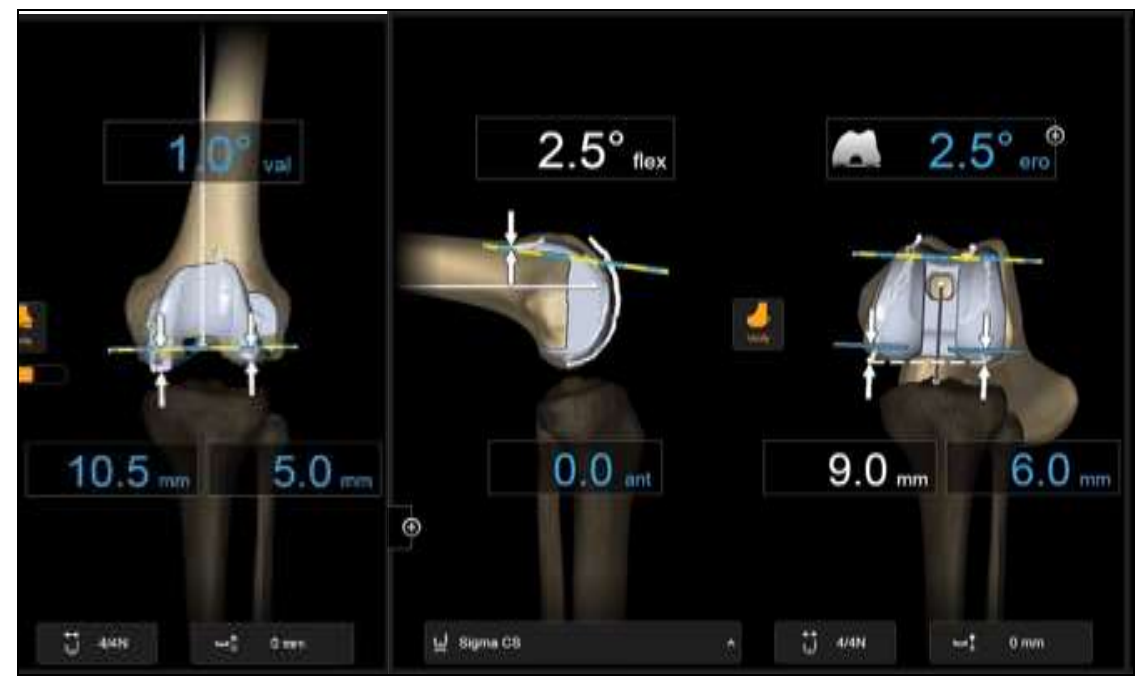

Fig 5: Femoral component alignment set for gap balance

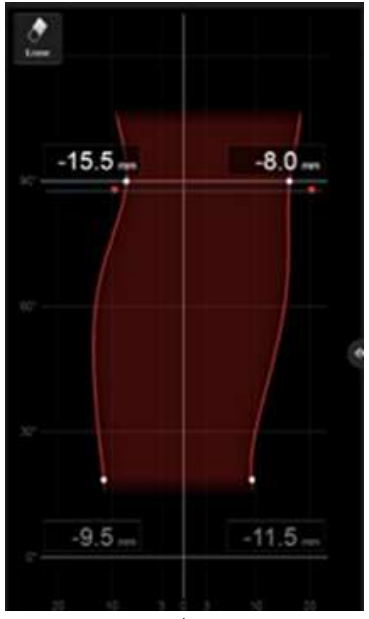

A

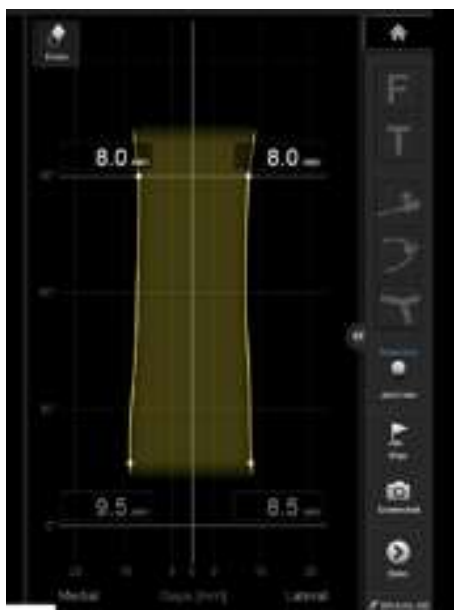

B

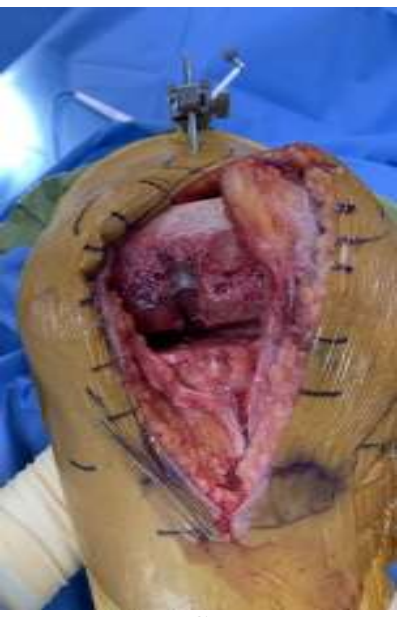

C

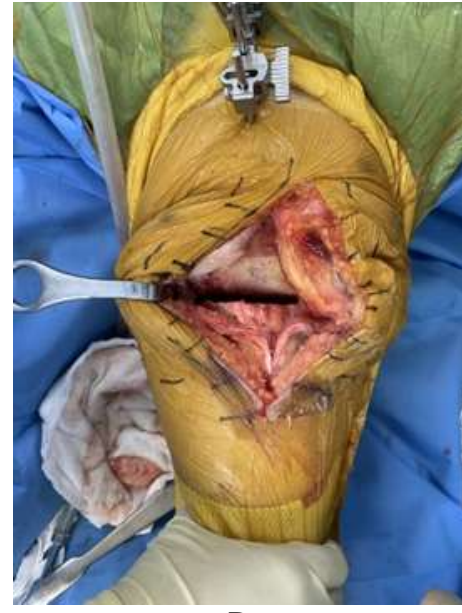

D

Fig 6: A) A narrow joint space is shown by CAS, due to severe bone loss in the posteromedial section; B) After augmentation with the metaphyseal sleeve, combined gab balance, and measured resection, a symmetrical flexion and extension gap was achieved with approximately $12-13.5 \mathrm{~mm}$ joint space ( $8-9.5 \mathrm{~mm}$, plus a tibial tray thickness of $4 \mathrm{~mm})$. C, D) Intraoperative flexion and extension gap.

The final implant was changed to an unconstrained total knee prosthesis; PFC Sigma PS femur size 4, M.B.T revision tray, $45 \mathrm{~mm} \mathrm{M/L}$ metaphyseal sleeve with tibial stem, $10 \mathrm{~mm}$ polyethylene insert (DePuy).

The patient was discharged on day four after surgery, and followed-up at two weeks, one and three months. Her range of motion (ROM) was established at $0^{\circ}-130^{\circ}$ (Figure 8) using the preoperative KSS index: KSS-knee and KSSfunction indices were 0 and 5, respectively. Postoperatively, these had improved to 91 (KSS-knee) and 85 (KSSfunction). Postoperative radiograph shows well fit prosthesis with good alignment: medial proximal tibial angle (MPTA) $90^{\circ}$, medial distal femoral angle (MDFA) $95^{\circ}$ and posterior distal femoral angle (PDFA) $88^{\circ}$ 


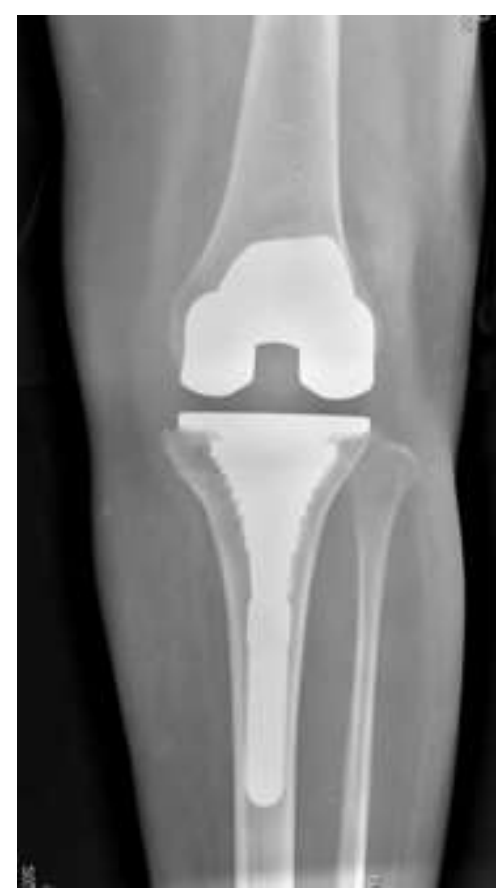

A

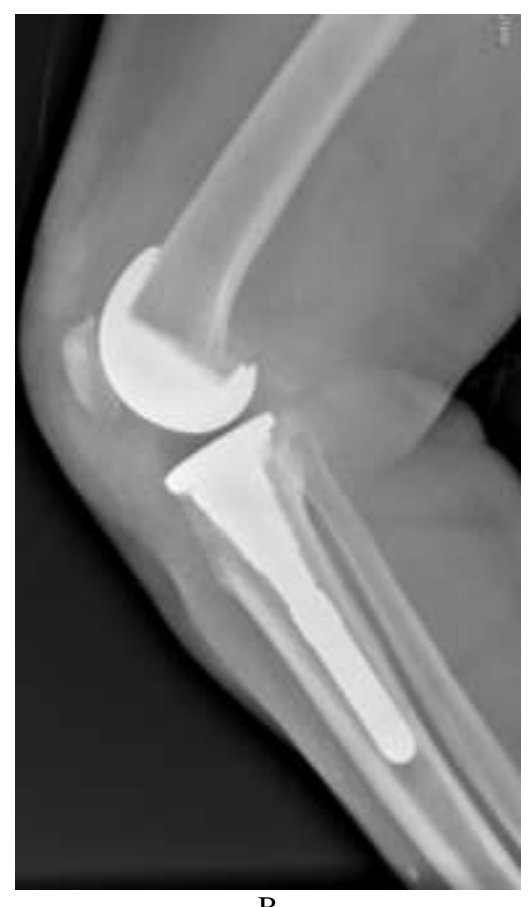

B

Fig 7: Postoperative radiographs A) AP view, B) Lateral view.

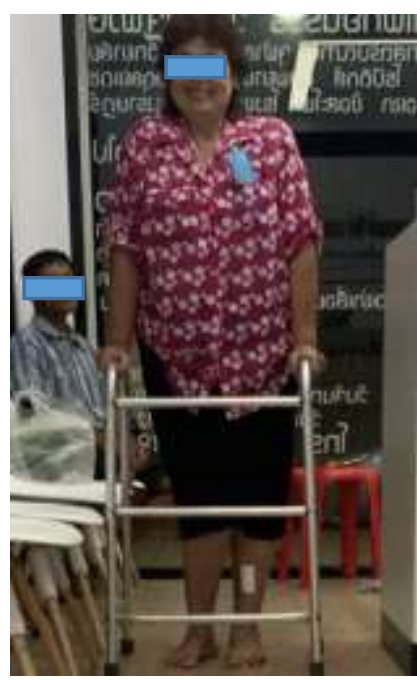

A

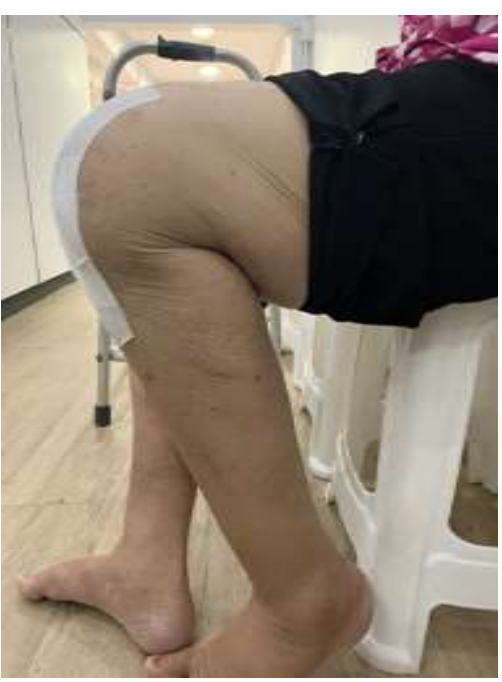

B

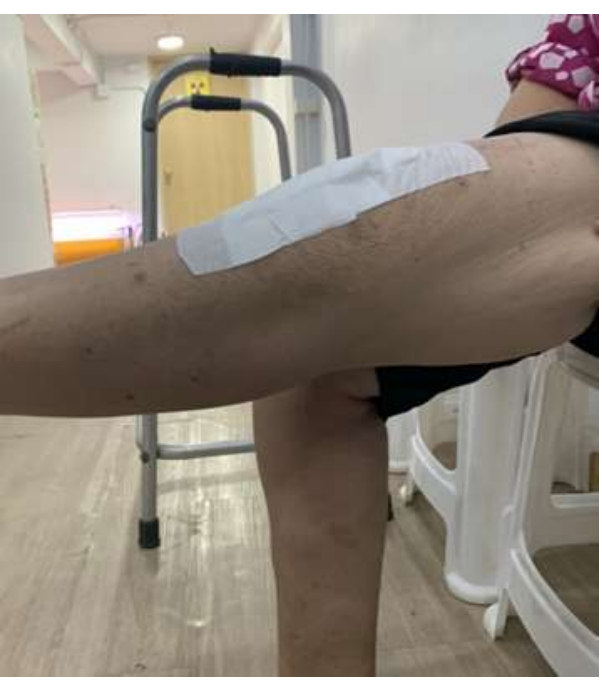

C

Fig 8: A) Postoperative alignment B, C) Postoperative ROM at two weeks

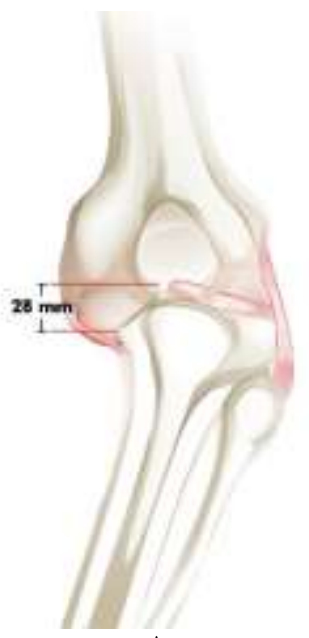

A

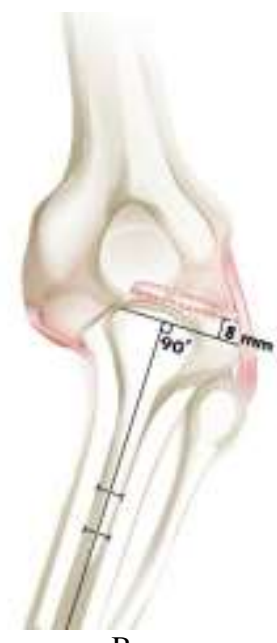

B

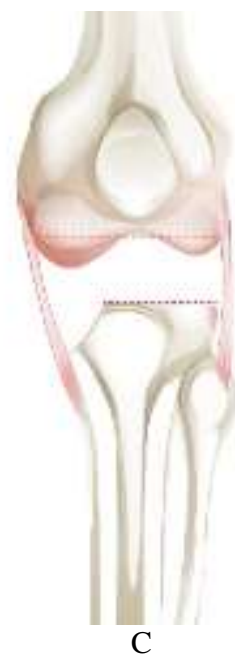

C 

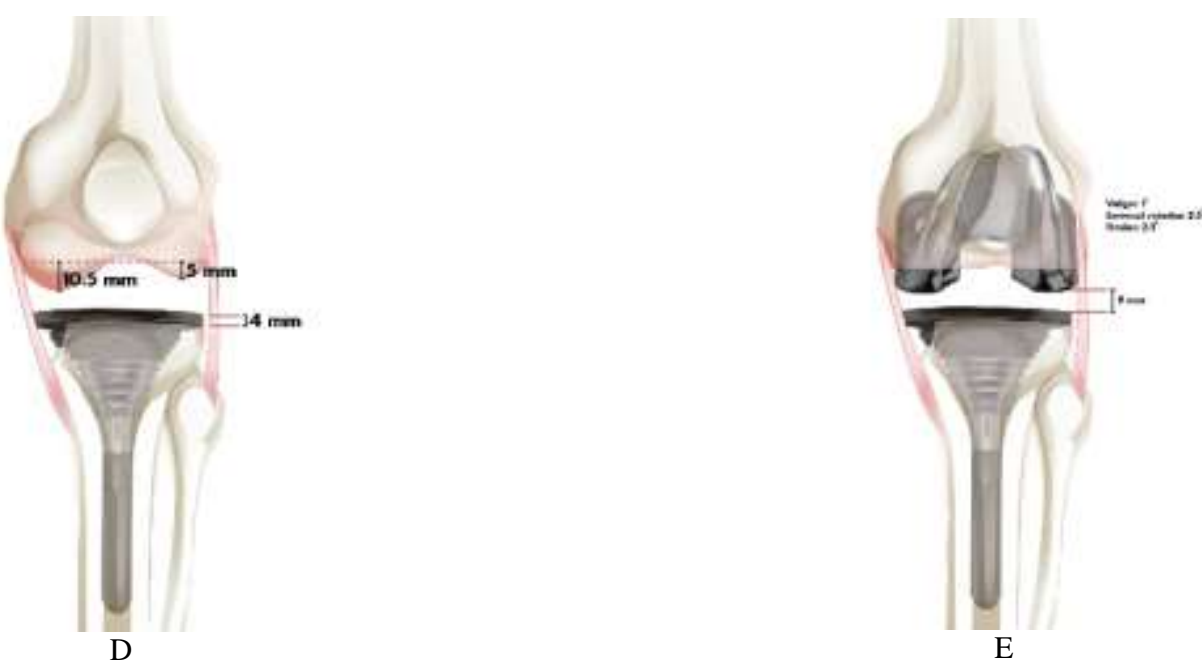

Figure 9: Summary of techniques, A) bone defects and alignment, B) reference point (lateral plateau $8 \mathrm{~mm}$ ), C \& D) correction of bone defect by metaphyseal sleeve, which was re-registered, E) femoral implant position was facilitated by measuring resection and minimally adjusting CAS.

\section{Discussion and literature review}

CAS significantly improves the accuracy and precision of component alignment for total knee arthroplasty. The approach is clinically superior with long-term functional outcomes, however revision rates are still debated in the literature. In their meta-analysis, Van der List et al. systematically reviewed 474,197 patients from a 2014 Australian registry, a 2011 Norwegian registry, and a 2014 New Zealand registry. These authors observed no differences in annual revision rates using computer navigation for total knee arthroplasty, versus conventional surgery ${ }^{[7]}$. Similarly, Jones et al. reported no conclusive outcomes for revision rates ${ }^{[8]}$. Recently, Rhee et al. reported no differences in survivorship between 1,348 navigation and 1,347 conventional total knee arthroplasties in their meta-analysis of randomized controlled trials ${ }^{[9]}$.

While several reports have demonstrated no clinical superiority in outcomes and revision rates for total knee arthroplasty ${ }^{[7,8]}$, no studies have yet shown inferiority data for CAS. Data from the previous studies predominantly reported from the USA only $6.7 \%$ of patient underwent CAS/robotic surgery (AJRR, 2019) ${ }^{[10]}$.

Recent evidence from the Australian Orthopedic Association and National Joint Replacement Registry has shown that CAS procedures for primary total knee arthroplasty increased from $2.4 \%$ in 2003 to $33.2 \%$ in 2019 . In CAS groups, including 114,859 navigate total knee arthroplasties, the 10-year revision rate in patients less than 65 -years-old, and component loosening for overall age was significantly lower than conventional surgery ${ }^{[11]}$.

We hypothesize that despite component alignment and good functional outcomes, other predisposing factors can often determine outcomes in individual patients, such as surgeon volume and training status ${ }^{[12]}$, or in complex primary knee, which increased reoperation and revision rates associated with the use of constrained implants ${ }^{[1]}$.

A recent CAS study showed significant benefits in correcting extra-articular deformities ${ }^{[13]}$, but no reports have yet outlined the use of CAS in a complex primary case, with an intra-articular bone defect. One potential reason for this is the difficulty in defining accurate anatomical references. In our case study, we solved the anatomical landmark limitation issue by choosing the lateral tibial joint line as the primary reference for tibial bone resection. The bone defect was corrected by tibial trial with or without stem, and gap balancing was performed after we re-registered the navigation to improve overall accuracy. In this complex primary case, ligament balance and stability were ultimately generated using an unconstrained prosthesis.

\section{Conclusion}

Our CAS approach allows for the easy correction of intraarticular knee deformity and ligament balancing. The procedure can be faithfully reproduced by surgeons. We believe that CAS combined with a conventional technique will help surgeons improve; accuracy, component precision, and ligament balance in complex primary knee arthroplasty. This strategy may be associated with a reduction in the use of a constrained prosthesis, and improved long-term outcomes.

\section{Acknowledgements}

The author would like to thank Assist. Prof. AVM. Chumroonkiet Leelasestaporn, Sqn. Ldr. Win Boonsirikumchai for clinical case discussion. The study was conducted without external sources of funding.

\section{Conflict of interest statement}

The authors report no conflicts of interest.

\section{References}

1. Baldini A, Castellani L, Traverso F, Balatri A, Balato G, Franceschini V. The difficult primary total knee arthroplasty: A review. Bone Joint J 2015;97-B(10 Suppl A):30-9.

2. Martin JR, Beahrs TR, Stuhlman CR, Trousdale RT. Complex primary total knee arthroplasty: long-term outcomes. J Bone Joint Surg Am 2016;98:1459-70.

3. Blakeney WG, Khan RJ, Wall SJ. Computer-assisted techniques versus conventional guides for component alignment in total knee arthroplasty: A randomized controlled trial. J Bone Joint Surg Am 2011;93:137784.

4. Matziolis G, Krocker D, Weiss U, Tohtz S, Perka C. A prospective, randomized study of computer-assisted and conventional total knee arthroplasty: three-dimensional 
evaluation of implant alignment and rotation. J Bone Joint Surg Am 2007;89:236-43.

5. Decking R, Markmann Y, Fuchs J, Puhl W, Scharf HP. Leg axis after computer-navigated total knee arthroplasty: A prospective randomized trial comparing computer-navigated and manual implantation. $\mathbf{J}$ Arthroplasty 2005;20:282-8.

6. Hetaimish BM, Khan MM, Simunovic N, Al-Harbi HH, Bhandari M, Zalzal PK. Meta-analysis of navigation vs conventional total knee arthroplasty. J Arthroplasty 2012;27:1177-82.

7. Van der List JP, Chawla H, Joskowicz L, Pearle AD. Current state of computer navigation and robotics in unicompartmental and total knee arthroplasty: A systematic review with meta-analysis. Knee Surg Sports Traumatol Arthrosc 2016;24:3482-95.

8. Jones CW, Jerabek SA. Current role of computer navigation in total knee arthroplasty. J Arthroplasty 2018;33:1989-93.

9. Rhee SJ, Kim HJ, Lee CR, Kim CW, Gwak HC, Kim JH. A comparison of long-term outcomes of computernavigated and conventional total knee arthroplasty. J Bone Joint Surg Am 2019;101:1875-85.

10. American Joint Replacement Registry (AJRR). American Academy of Orthopedic Surgeons 2019 annual report. https://connect.ajrr.net/hubfs/PDFs\%20and\%20PPTs/A AOS_AJRR_2019_Annual_Report_Update_FINAL_15 0DPI.pdf?hsCtaTracking $=1 \mathrm{~d} 80 \mathrm{e} 9 \mathrm{fa}-66 \mathrm{fe}-4525-\mathrm{a} 5 \mathrm{cb}-$ 15c30ec19a1f\%7C8becda93-a51e-41cc-bcfaca9130c1add6 21 November, 2020.

11. Australian Orthopaedic Association National Joint Replacement Registry (AOANJRR). Hip, knee, and shoulder arthroplasty: Annual Report 2019. https://aoanjrr.sahmri.com/documents/10180/668596/Hi p\%2C+Knee+\%26+Shoulder+Arthroplasty/c287d2a322df-a3bb-37a2-91e6c00bfcf0 6 July, 2020.

12. Kazarian GS, Lawrie CM, Barrack TN et al. The impact of surgeon volume and training status on implant alignment in total knee arthroplasty. J Bone Joint Surg Am 2019;101:1713-23.

13. Khanna V, Sambandam SN, Ashraf M, Mounasamy V. Extra-articular deformities in arthritic knees-a grueling challenge for arthroplasty surgeons: an evidence-based update. Orthopedic Reviews 2018;9:124-35. 